\title{
Basal-pyramidal dislocation lock in deformed magnesium
}

\author{
B. Li $^{{ }^{1 *}}$, Q.W. Zhang ${ }^{1}$, S.N. Mathaudhu ${ }^{2}$ \\ ${ }^{1}$ Department of Chemical and Materials Engineering, University of Nevada, Reno, NV 89557, USA \\ ${ }^{2}$ Department of Mechanical Engineering, University of California, Riverside, CA 92521, USA \\ * Corresponding author: Bin Li, Email: binl@unr.edu; Tel: 775-784-4507
}

\begin{abstract}
Dislocations and stacking faults in unalloyed magnesium processed by equal-channelangular-extrusion are investigated by transmission electron microscopy. Stacking faults as wide as hundreds of nanometers and complex dislocation configurations are revealed, which creates diffraction contrast that is challenging to interpret. To elucidate the dislocation interaction, atomistic simulations are conducted. The concomitant results show that the leading partial dislocations on the basal and the pyramidal planes interact and form a dislocation lock, creating the diffraction contrast observed in experiments. The dislocation locks may act as obstacles to dislocation glide and contribute to hardening.
\end{abstract}

Key words: Magnesium; Dislocation; Stacking fault; Transmission electron microscopy.

(C) 2017. This manuscript version is made available under the Elsevier user license http://www.elsevier.com/open-access/userlicense/1.0/ 
Magnesium and it's alloys are being considered for a wide variety of application due to their light weight, high specific strength and availability, however broader application is limited by the low ductility at room temperature [1]. Compared to aluminum (Al) alloys that have facecentered-cubic (fcc) crystal structures, hexagonal-close-packed (hcp) magnesium ( $\mathrm{Mg}$ ) respond to mechanical loading very differently, in part due to complex dislocation structures, and in part due to deformation twinning [2]. In terms of dislocation slip, three different slip planes have been reported in the literature: basal, prismatic and pyramidal systems [3]. The basal dislocation tends to dissociate into two Shockley partials, i.e. $\frac{1}{3}\langle 2 \overline{1} \overline{1} 0\rangle \rightarrow \frac{1}{3}\langle 10 \overline{1} 0\rangle+S F+\frac{1}{3}\langle 1 \overline{1} 00\rangle$, and a stacking fault (SF) is created between the two partials. Although the basal stacking fault energy (SFE) of $\mathrm{Mg}$ is low $\left(25 \sim 35 \mathrm{~mJ} / \mathrm{m}^{2}\right)$, the equilibrium split distance between the Shockley partials is only $1 \sim 3 \mathrm{~nm}$ [4]. Thus, in conventional transmission electron microscopy (TEM) observations, the basal dislocations usually appear to be non-dissociated [5]. Previous reports also reveal distinct basal SFs which are frequently observed inside $\{10 \overline{1} 2\}\langle 10 \overline{1} \overline{1}\rangle$ deformation twins [6-9]. These SFs are generated by incoherent twin boundary migration, and no partial dislocations that bound the SFs are involved [6]. Prismatic dislocations are often observed in deformed polycrystalline $\mathrm{Mg}$ in TEM analyses [10,11], but their dissociation is more complex than basal dislocations due to the corrugated nature of the slip plane. Recently, Liao et al. [12] uncovered the unstable dissociation of a prismatic dislocation. Due to the possibly high SFE on the prismatic plane, the SF was quickly eliminated by the trailing partial. Thus, prismatic dislocations also appear to be full dislocation lines in TEM.

Pyramidal slip in hep metals has been reported by a number of researchers [13-20]. Two possible pyramidal slip planes were reported: $\{10 \overline{1} 1\}[13,20-23]$ and $\{11 \overline{2} 2\}[14,15,24]$. The Burgers vector, $\frac{1}{3}\langle 11 \overline{2} \overline{3}\rangle$, i.e. the $\langle c+a\rangle$, equals $a \cdot \sqrt{1+\gamma^{2}} \approx 0.612 \mathrm{~nm}$ for $\operatorname{Mg}(a$ is the lattice 
parameter $0.321 \mathrm{~nm}$ and $\gamma$ the $c / a$ ratio 1.624) is fairly large if compared to other Burgers vectors in pure metals, and this has prompted a number of atomistic simulations to investigate possible dissociations and the resultant SFs [16-23,25,26]. Recently, Wu and Curtin [25] simulated dissociation of a $\{11 \overline{2} 2\}\langle 11 \overline{2} \overline{3}\rangle$ pyramidal II dislocation, using a modified embedded atom method (MEAM) [27] potential and long-time atomistic simulations. They revealed basaldissociated immobile structures that account for the high hardening and low ductility in $c$-axis compression.

Due to the complexity, dislocation structures, especially their interactions in hcp Mg have not been thoroughly understood, and the resulting incongruity over slip activity in terms of mechanism and dislocation interactions necessitates further investigation. The purpose of this work is to carefully probe dislocation and SF activities in unalloyed Mg processed by equalchannel-angular-extrusion (ECAE) [28]. Complex diffraction contrast is observed, thus concurrent atomistic simulations are performed to facilitate interpretation of the image contrast. This combination of TEM and atomistic simulations enables unraveling of the complex dislocation interactions in hep metals.

The starting material was from an as-cast $99.9 \%$ purity Norsk Hydro ingot, courtesy of Magnesium Elektron - North America (Madison, IL, USA). Its columnar grains had diameters of $\sim 1.0 \mathrm{~mm}$ and lengths on the order of tens of millimeters. No thermomechanical homogenization pre-processing was done prior to ECAE processing. Rods of $12.5 \mathrm{~mm}$ diameter and $50 \mathrm{~mm}$ long were processed via route $\mathrm{C}\left(90^{\circ}\right.$ channel intersection, $180^{\circ}$ rotation about the rod long axis between passes [28]) at $150^{\circ} \mathrm{C}$ up to four passes, at a punch speed of $2.54 \mathrm{~mm} / \mathrm{s}$ without applied backpressure. TEM specimens were prepared by polishing samples to a nominal thickness of 130 $\mu \mathrm{m}$ using a series of sand paper down to grit number 800 . Three millimeter discs were then 
mechanically punched from the sample. Specimens were then polished using a Tenupol-3 electropolisher with an electrolyte $(0.5 \%$ perchloric acid $+20 \%$ nitric acid + ethanol $)$. The specimens were then cleaned by ion-milling for about 0.5 hours with liquid nitrogen cooling and very gentle milling conditions (low incidental angle and low voltage). TEM observations were carried out on a Philips 420 microscope with a double-tilt specimen stage, and an accelerating voltage of $120 \mathrm{kV}$. After four passes of ECAE, the grain structure was significantly refined with an average grain size of $3.0 \mu \mathrm{m}$. The overall microstructure is inhomogeneous with regions of fine grains from dynamic recrystallization and other regions of relatively large grains which are heavily deformed but not recrystallized.

To further elucidate our experimental observations, atomistic simulations were performed which predictively model the interaction between the basal slip and the pyramidal dislocations and the resultant SFs. The MD simulations were carried out with a box size of $40 \times 40 \times 40 \mathrm{~nm}$ containing about 2.8 million $\mathrm{Mg}$ atoms. Embedded atom method (EAM) potentials [29,30] for Mg-Al alloy systems were used in our simulations. These potentials were well developed by Liu et al. [31] and have been used in numerous atomistic simulations of $\mathrm{Mg}$ and $\mathrm{Mg}$ alloys $[20,32,33]$. The lattice was oriented such that a shear strain was applied parallel to the $\{10 \overline{1} 2\}$ plane and along the $\langle 10 \overline{1} \overline{1}\rangle$ direction. The strain rate was about $10^{8} / \mathrm{sec}$. No periodic boundary condition was applied such that heterogeneous nucleation of dislocations at free surfaces was enabled. Constant temperature at $10 \mathrm{~K}$ was maintained throughout the simulation. No dislocations were pre-constructed. During shear deformation, slip systems on the basal, prismatic and pyramidal planes all can be activated, and interactions between these slip systems can be investigated. 
Figure 1a shows an array of stacking faults (SFs) in a specimen after two passes at $150^{\circ} \mathrm{C}$. The SFs are quite well-defined with visible bright/dark fringes in the bright-field micrograph. The fringes are similar to what are typically observed in fcc crystals [34], but the dark/bright contrast disrupts at some locations and no longer becomes complementary. This change in the contrast indicates that overlapping SFs are present [34]. At the ends of the SFs, dark contrast typical of dislocations can be observed, as indicated by the block arrow. Figure $1 \mathrm{~b}$ displays a weak-beam-dark-field (WBDF) image which confirms the presence of the overlapping SFs. As indicated by the block arrows, two SFs overlap with each other.

The contrast of the SFs dramatically changes when the specimen is tilted to the $\langle 2 \overline{1} \overline{1} 0\rangle$ zone axis. During tilting, the well-defined fringes gradually lose the bright/dark characteristic features, as shown in Figure 2a. When the zone axis $\langle 2 \overline{1} \overline{1} 0\rangle$ is reached, the fringes of SFs disappear and the contrast becomes straight lines decorated with dark contrast. Close examination reveals that each of these lines is made up of a number of individual segments (indicated by the red arrows) that are well aligned parallel to the trace of the basal plane. Meanwhile, dislocation lines that are parallel to the trace of the basal plane can be observed, as indicated by the black arrows. Because the electron beam is parallel to the basal plane of the specimen, these dislocation lines can be identified as basal dislocations. To identify the Burgers vector of the defects Figure $1 \mathrm{a}, 1 \mathrm{~b}$ and 2a, we performed WBDF imaging and the result is shown in Figure $2 \mathrm{~b}$. With $g=0002$, the features show a strong $\langle c\rangle$ component.

The presence of the dislocation contrast at the ends of the SFs (Figure 1a) indicates that the formation mechanism of these SFs differs from the non-equilibrium basal SFs observed inside deformation twins [6-9]. Zhang et al. recently showed that SFs that may cross a whole twin are not generated by Shockley partial dislocations, but rather, by incoherent twin boundary migration 
[6]. On the other hand, the width of the SFs in Figure 1 are as wide as hundreds of nanometers, several orders of magnitude wider than that of the equilibrium width of basal SFs generated by the split of a basal dislocation (1 3 nm [35]). These facts suggest that the SFs in Figure 1 are likely non-basal SFs. We can also exclude the possibility that these SFs are generated by prismatic slip because the split distance between the partial dislocations of prismatic slip is very small $(<1.0 \mathrm{~nm})$ [12]. Intriguing questions arise from these TEM observations: if the SFs are indeed non-basal, how the contrast of the straight lines (Figure $2 b$ ) parallel to the trace of the basal plane is produced? If the straight lines are associated with the basal slip, why they present a strong $\langle c\rangle$ component in the Burgers vector analysis?

In the following, we show atomistic simulation results that reveal basal and pyramidal dislocation reaction. Figure 3a shows a 2-D snapshot of the dislocations that were activated during shear deformation. In this plot, common neighbor analysis was used to display the defect structures [36]. Atoms at the perfect hcp lattice points are shown as red spheres. Two different types of dislocations can be identified: basal slip with the resultant basal SFs are displayed in green; and $\{10 \overline{1} 1\}$ pyramidal slip with the resultant SFs are displayed in gray. The trailing partials are not nucleated yet and only the leading partials can be seen. All the slip systems were activated near the corners of the single crystal due to the stress concentration at these locations. Near the bottom surface, the leading partial dislocations are indicated by the dark blue arrows. The Shockley partial of the basal slip has a Burgers vector of $\frac{1}{3}[10 \overline{1} 0]_{(0002)}$. Figure $3 b$ shows the Burgers circuit analysis for the $\{10 \overline{1} 1\}$ pyramidal slip. It can be seen that the leading partial of the pyramidal slip has a Burgers vector of $\frac{1}{4}[10 \overline{1} \overline{2}]_{\{10 \overline{1} 1\}}$. The two leading partials have opposite signs, so they attract rather than repel each other, as indicated by yellow arrows near the top surface. When the two dislocations eventually meet, the two SFs intersect and both 
dislocations become immobile after intersection. Hence, they are interlocked by the dislocation reaction. This scenario is similar to the Lomer-Cottrell lock [37,38] in which two mobile Shockley partials gliding on two different $\{111\}$ planes meet and combine into an immobile partial. The basal and the pyramidal dislocation reaction can be described as:

$$
\frac{1}{3}[10 \overline{1} 0]-\frac{1}{4}[10 \overline{1} \overline{2}] \rightarrow \frac{1}{12}[10 \overline{1} 0]+\frac{1}{2}[0001]
$$

This dislocation reaction creates a new Burgers vector that has two components. One component $\frac{1}{12}[10 \overline{1} 0]$ lies on the basal plane, and the other component $\frac{1}{2}[0001]$ lies perpendicular to the basal plane. Thus, a sessile dislocation is created because it is neither on the (0002) basal plane nor the $\{10 \overline{1} 1\}$ pyramidal plane. We define such a dislocation reaction as "basal-pyramidal dislocation lock".

To better resolve the dislocation interactions, we provide 3-D plots of the dislocation reaction and the SFs in Figure 4a and 4b. In Figure 4a, all the red atoms that are in the perfect hcp lattice points are removed. Also removed are the top and the bottom surfaces. Thus, only those atoms at the dislocation core and the SFs are shown. The configuration is slightly tilted to show the 3-D view of the dislocations and SFs. Near the bottom, the $\{10 \overline{1} 1\}$ pyramidal leading partial dislocation and the basal leading partial dislocation are gliding toward each other (indicated by the red arrows). Near the top surface, the basal SF and the pyramidal SF have impinged. The basal leading partial dislocation and the pyramidal leading partial dislocation react to form a basal-pyramidal lock. As a result, both slip systems becomes inactive. Figure $4 \mathrm{~b}$ shows a sessile dislocation line is created along the intersection between the basal and the $\{10 \overline{1} 1\}$ pyramidal plane.

The wide SFs and the complex diffraction contrast associated with the SFs and the involved dislocations observed in this work (Figure 1-2) and in previous reports in TEM [15,39-41], 
which have been difficult to interpret, can now be better understood from the dislocation reaction between basal slip and pyramidal slip revealed in our atomistic simulations. Although basal slip has the lowest critical stress in Mg [2,42,43], non-basal slip can also be activated where local stress concentrations exist, for example, grain boundaries where compatibility strain is required, dislocation cell structures and dislocation pile-ups [5]. Given the large shear strain in the ECAE processing ( $\sim 1.15$ per pass), pyramidal slip systems can be activated. TEM shows that the leading partial dislocation of the $\{10 \overline{1} 1\}$ pyramidal slip creates a wide SF (Figure 1a), but the nucleation of the trailing partial dislocation is usually delayed due to the asymmetric nature of the energy landscape on the pyramidal plane [20]. The trailing partial has a larger Burgers vector and is more difficult to nucleate [20]. Therefore, these wide SFs on the $\{10 \overline{1} 1\}$ pyramidal planes can be readily intersected by basal slip which is the easiest to activate. As the leading partials of the basal and the pyramidal slip react to form the sessile lock, an immobile dislocation line with a Burgers vector which has both $\langle c\rangle$ and $\langle a\rangle$ components (Equ.1) is produced. Such a dislocation line lies along the intersection between the basal and the $\{10 \overline{1} 1\}$ pyramidal plane. In TEM observation, if the specimen is tilted such that the electron beam is parallel to the basal plane, this sessile dislocation will produce a straight line parallel to the trace of the basal plane, as observed by numerous researchers [15,39-41]. However, the sessile dislocation has a $\langle c\rangle$ component. If we perform Burgers vector analysis with $g=0002$ in the visibility criterion $(g \cdot b \neq 0)$, a strong diffraction contrast from the $\langle c\rangle$ component can be observed (Figure $2 b)$. If we carefully tilt the TEM specimen to proper diffraction condition, we can observe very wide SFs (Figure 1a) which come from the locked pyramidal slip. If we tilt the TEM specimen such that the dark-bright fringes of the SFs disappear, we can only observe the basal dislocations and the resultant dark contrast from the basal dislocations (Figure 2a). Because the equilibrium width 
of the basal SFs is only a couple of nanometers, it is difficult to resolve the basal SFs at low magnifications. Thus, the basal dislocations usually appear to be non-dissociated in TEM. As such, the intriguing, complex diffraction contrast can be plausibly explained.

Finally, dislocation reactions may strongly influence the hardening behavior of hcp metals in deformation. Bertin et al. [44] analyzed potential dislocation reactions between basal, prismatic and $\{11 \overline{2} 2\}\langle 11 \overline{2} \overline{3}\rangle$ pyramidal slip, and suggested that interaction between $\langle a\rangle$ and $\langle c+a\rangle$ leads to sessile junctions, contributing to hardening. The work by Sandlöbes et al. [45] suggests that, as the SFE is decreased by solute atoms, the SFs act as a heterogeneous source for pyramidal dislocations. As a result, the ductility of $\mathrm{Mg}$ alloys can be improved. Jian et al. [46] show that superior strength can be obtained by introducing a high density SFs in a Mg alloy. The SFs impede dislocation slip, and enhance dislocation accumulation. Conceivably, the interlocked basal and pyramidal SFs could act as obstacles to dislocation glide, such as shown in TEM studies by Agnew et al. [47] that basal faults interact with pyramidal dislocations.

To conclude, we performed TEM observations of dislocation structures in pure Mg processed by ECAE. Wide, overlapping SFs with complex diffraction contrast were observed. In parallel, atomistic simulations reveal that (0002) basal slip and $\{10 \overline{1} 1\}$ pyramidal slip interact, and that the leading partial dislocations react and create a sessile dislocation lock at the intersection of the two slip planes. The complex diffraction contrast observed in TEM analyses can be plausibly explained as a result of such interaction. This basal-pyramidal lock may impede the glide of dislocations, contributing to work hardening in $\mathrm{Mg}$ and $\mathrm{Mg}$ alloys.

\section{Acknowledgements}


The authors are grateful to Prof. K.T. Hartwig of Texas A\&M University for the ECAE

processing of the materials used in the study. BL gratefully thanks support from the U.S. National Science Foundation (NSF) under Grant \#1635088. SNM gratefully acknowledges support from NSF under Grant \#1554632.

\section{References}

[1] T.M. Pollock, Science 328 (2010) 986-987.

[2] M.M. Avedesian, H. Baker, ASM Specialty Handbook: Magnesium and Magnesium Alloys, ASM International, 1999.

[3] D.J. Bacon, V. Vitek, Metall. Mater. Trans. A 33 (2002) 721-733.

[4] J.A. Yasi, L.G. Hector Jr., D.R. Trinkle, Acta Mater. 58 (2010) 5704-5713.

[5] B. Li, E. Ma, K.T. Ramesh, Metall. Mater. Trans. A 39 (2008) 2607-2614.

[6] X. Zhang, B. Li, Q. Liu, Acta Materialia 90 (2015) 140-150.

[7] S.G. Song, G.T. Gray, Philos. Mag. A 71 (1995) 263-274.

[8] S.G. Song, G.T. Gray III, Acta Metall. Mater. 43 (1995) 2325-2337.

[9] S.G. Song, G.T. Gray III, Acta Metall. Mater. 43 (1995) 2339-2350.

[10] B. Li, S. Joshi, K. Azevedo, E. Ma, K.T. Ramesh, R.B. Figueiredo, T.G. Langdon, Mater. Sci. Eng. A 517 (2009) 24-29.

[11] B. Li, S.P. Joshi, O. Almagri, Q. Ma, K.T. Ramesh, T. Mukai, Acta Mater. 60 (2012) $1818-1826$.

[12] M. Liao, B. Li, M.F. Horstemeyer, Scr. Mater. 69 (2013) 246-249.

[13] H. Yoshinaga, R. Horiuchi, Transactions of the Japan Institute of Metals 4 (1963) 1-8.

[14] J. Koike, T. Kobayashi, T. Mukai, H. Watanabe, M. Suzuki, K. Maruyama, K. Higashi, Acta Mater. 51 (2003) 2055-2065.

[15] T. Obara, H. Yoshinga, S. Morozumi, Acta Metall. 21 (1973) 845-853.

[16] M.H. Liang, D.J. Bacon, Philos. Mag. A 53 (1986) 181-204.

[17] Y. Minonishi, S. Ishioka, M. Koiwa, S. Morozumi, Philos. Mag. A 46 (1982) 761-770.

[18] H. Numakura, Y. Minonishi, M. Koiwa, Philos. Mag. A 62 (1990) 525-543.

[19] J.R. Morris, J. Scharff, K.M. Ho, D.E. Turner, Y.Y. Ye, M.H. Yoo, Philos. Mag. A 76 (1997) 1065-1077.

[20] B. Li, E. Ma, Philos. Mag. 89 (2009) 1223-1235.

[21] J.A. Yasi, L.G. Hector Jr., D.R. Trinkle, Acta Mater. 60 (2012) 2350-2358.

[22] Y. Tang, J.A. El-Awady, Acta Mater. 71 (2014) 319-332.

[23] K.Y. Xie, Z. Alam, A. Caffee, K.J. Hemker, Scr. Mater. 112 (2016) 75-78.

[24] J. Geng, M.F. Chisholm, R.K. Mishra, K.S. Kumar, Philos. Mag. 95 (2015) 3910-3932.

[25] Z. Wu, W.A. Curtin, Nature 526 (2015) 62-67.

[26] H. Fan, J.A. El-Awady, Mater. Sci. Eng. A 644 (2015) 318-324.

[27] M.I. Baskes, S.G. Srinivasan, S.M. Valone, R.G. Hoagland, Phys. Rev. B 75 (2007) 94113. 
[28] M. Al-Maharbi, I. Karaman, I.J. Beyerlein, D. Foley, K.T. Hartwig, L.J. Kecskes, S.N. Mathaudhu, Mater. Sci. Eng. A 528 (2011) 7616-7627.

[29] M.S. Daw, M.I. Baskes, Phys. Rev. Lett. 50 (1983) 1285-1288.

[30] M.S. Daw, M.I. Baskes, Phys. Rev. B 29 (1984) 6443-6453.

[31] X.-Y. Liu, J.B. Adams, F. Ercolessi, J.A. Moriarty, Model. Simul. Mater. Sci. Eng. 4 (1996) 293.

[32] B. Li, E. Ma, Acta Mater. 57 (2009) 1734-1743.

[33] B. Li, M. Liao, Q. Ma, Z. McClelland, Comput. Mater. Sci. 101 (2015) 175-180.

[34] D.B. Williams, C.B. Carter, Transmission Electron Microscopy: A Textbook for Materials Science, 2nd edition, Springer, New York, 2009.

[35] J.A. Yasi, T. Nogaret, D.R. Trinkle, Y. Qi, L.G.H. Jr, W.A. Curtin, Model. Simul. Mater. Sci. Eng. 17 (2009) 55012.

[36] A. Stukowski, Model. Simul. Mater. Sci. Eng. 18 (2010) 15012.

[37] A.H. Cottrell, Lond. Edinb. Dublin Philos. Mag. J. Sci. 43 (1952) 645-647.

[38] W.M. Lomer, Lond. Edinb. Dublin Philos. Mag. J. Sci. 42 (1951) 1327-1331.

[39] J.F. Stohr, J.P. Poirier, Philos. Mag. 25 (1972) 1313-1329.

[40] B. Li, P.F. Yan, M.L. Sui, E. Ma, Acta Mater. 58 (2010) 173-179.

[41] S.R. Agnew, J.A. Horton, M.H. Yoo, Metall. Mater. Trans. A 33 (2002) 851-858.

[42] M.R. Barnett, Metall. Mater. Trans. A 34 (2003) 1799-1806.

[43] E.W. Kelley, W.F. Hosford Jr., Transactions of the Metallurgical Society of AIME 242 (1968) 654-661.

[44] N. Bertin, C.N. Tomé, I.J. Beyerlein, M.R. Barnett, L. Capolungo, Int. J. Plast. 62 (2014) $72-92$.

[45] S. Sandlöbes, M. Friák, S. Zaefferer, A. Dick, S. Yi, D. Letzig, Z. Pei, L.-F. Zhu, J. Neugebauer, D. Raabe, Acta Mater. 60 (2012) 3011-3021.

[46] W.W. Jian, G.M. Cheng, W.Z. Xu, H. Yuan, M.H. Tsai, Q.D. Wang, C.C. Koch, Y.T. Zhu, S.N. Mathaudhu, Mater. Res. Lett. 1 (2013) 61-66.

[47] S.R. Agnew, L. Capolungo, C.A. Calhoun, Acta Mater. 82 (2015) 255-265. 

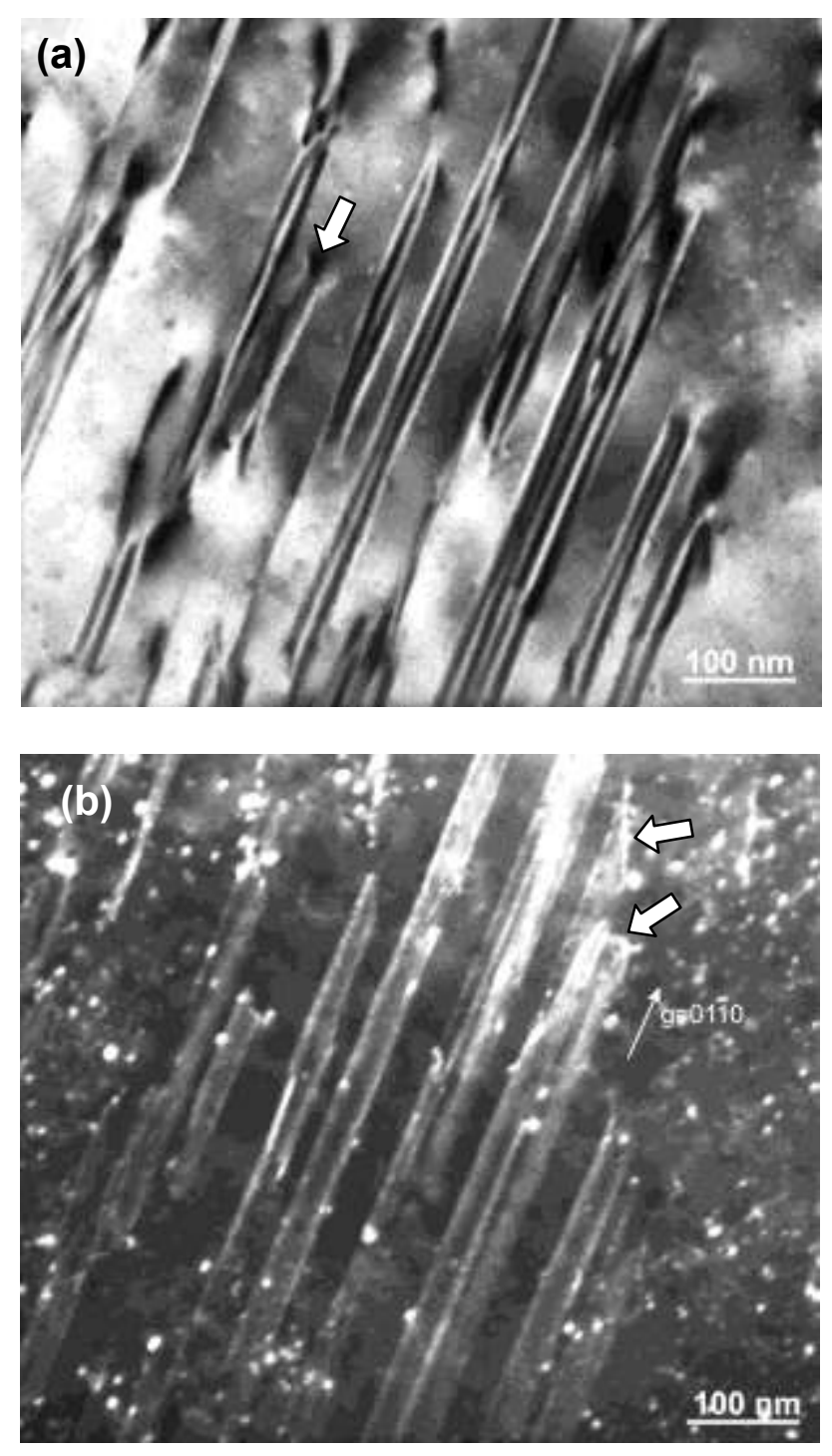

Figure 1. (a) Bright/dark fringes of SFs with a high density were observed in a specimen after two passes via route $\mathrm{C}$. The widths of the SFs are on the order of hundreds of nanometers. Locations where the dark/bright contrast disrupts indicate that SFs are overlapping. The dark contrast at the end of the SF (denoted by the block arrow) indicates the presence of a partial dislocation. (b) Weak-beam-dark-field (WBDF) image clearly shows the overlapping SFs (indicated by the block arrows). 

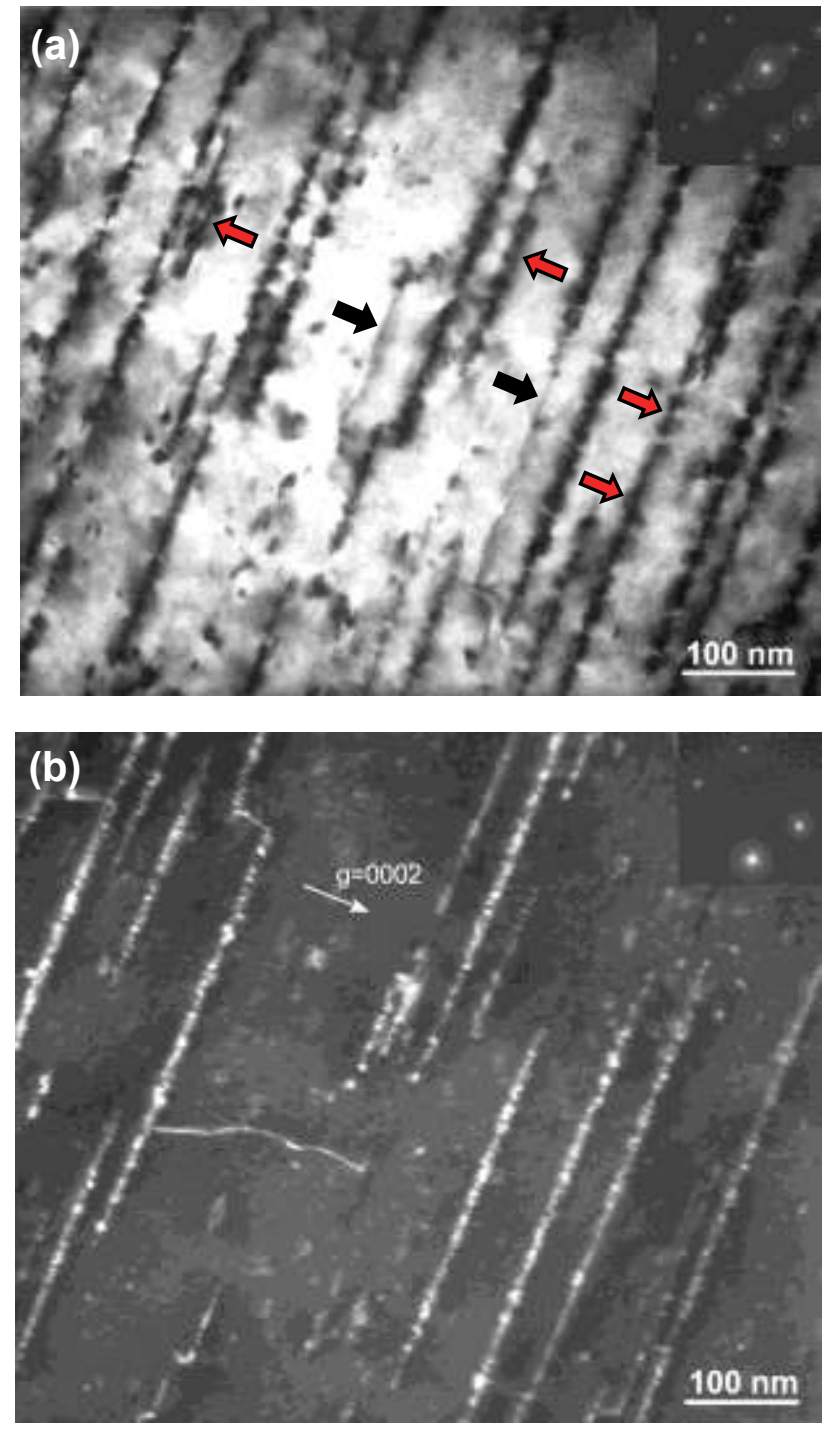

Figure 2. When the specimen was tilted to the $\langle 2 \overline{1} \overline{1} 0\rangle$ zone axis, the bright/dark fringes of SFs become "straight lines" decorated with dark speckles. Close examination reveals that the lines are actually dislocations (indicated by the block arrows) lying on the basal planes. (b) Weak beam dark field image $(\mathrm{g}=0002)$ of the dislocation configurations in (a). A $\langle c\rangle$ component in the Burgers vector can be seen. 


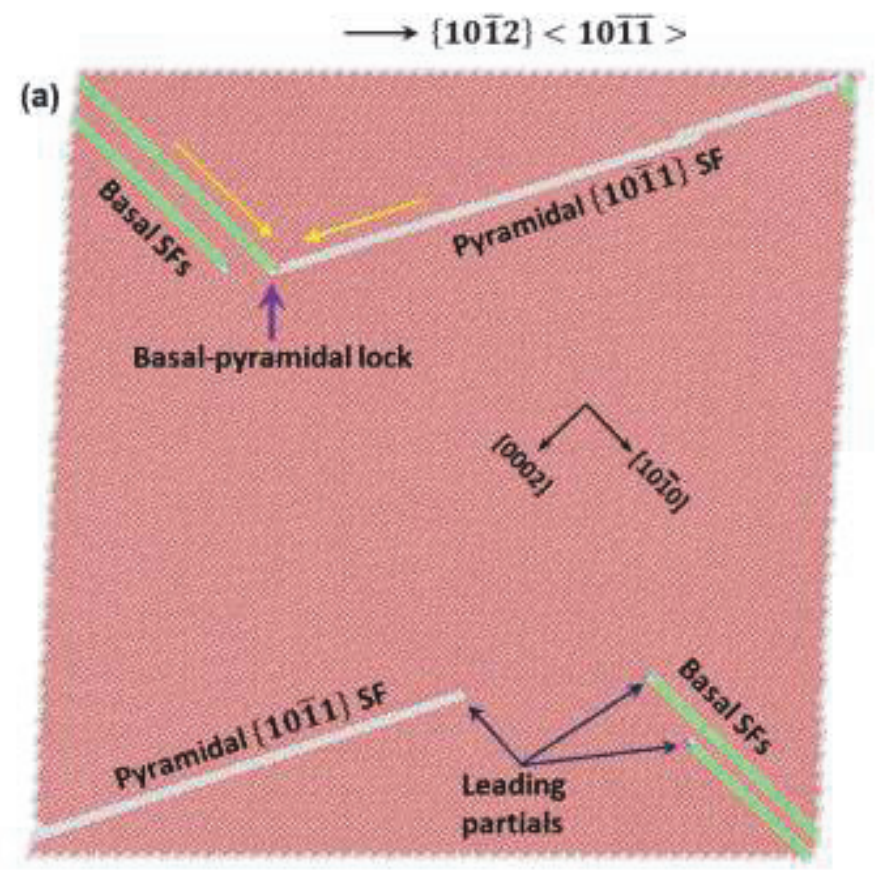

(b)

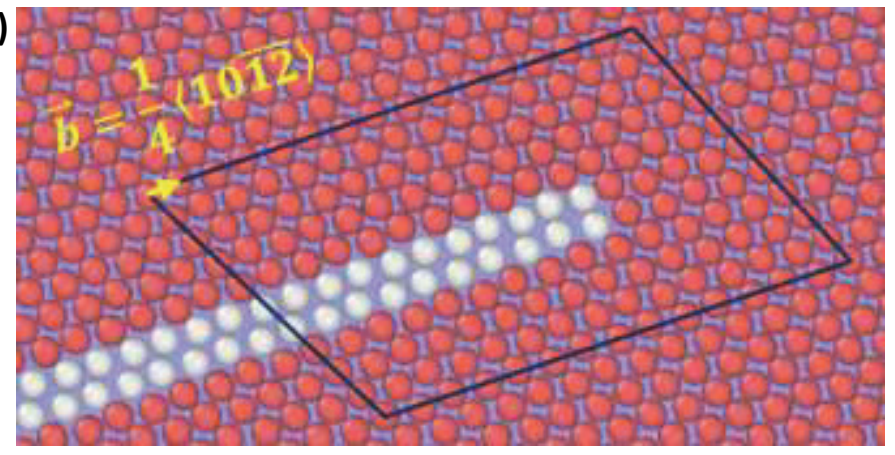

Figure 3. (a) Atomistic simulations of defect activities during shear deformation. Basal and pyramidal slip are activated. The basal stacking fault (SF in green) and the pyramidal (SF in gray) intersect (indicated by the purple arrow), and both slip systems are locked and become immobile. (b) Burgers circuit analysis indicates that the Burgers vector of the leading partial of the pyramidal slip equals $\frac{1}{4}\langle 10 \overline{1} \overline{2}\rangle$ (indicated by the yellow 

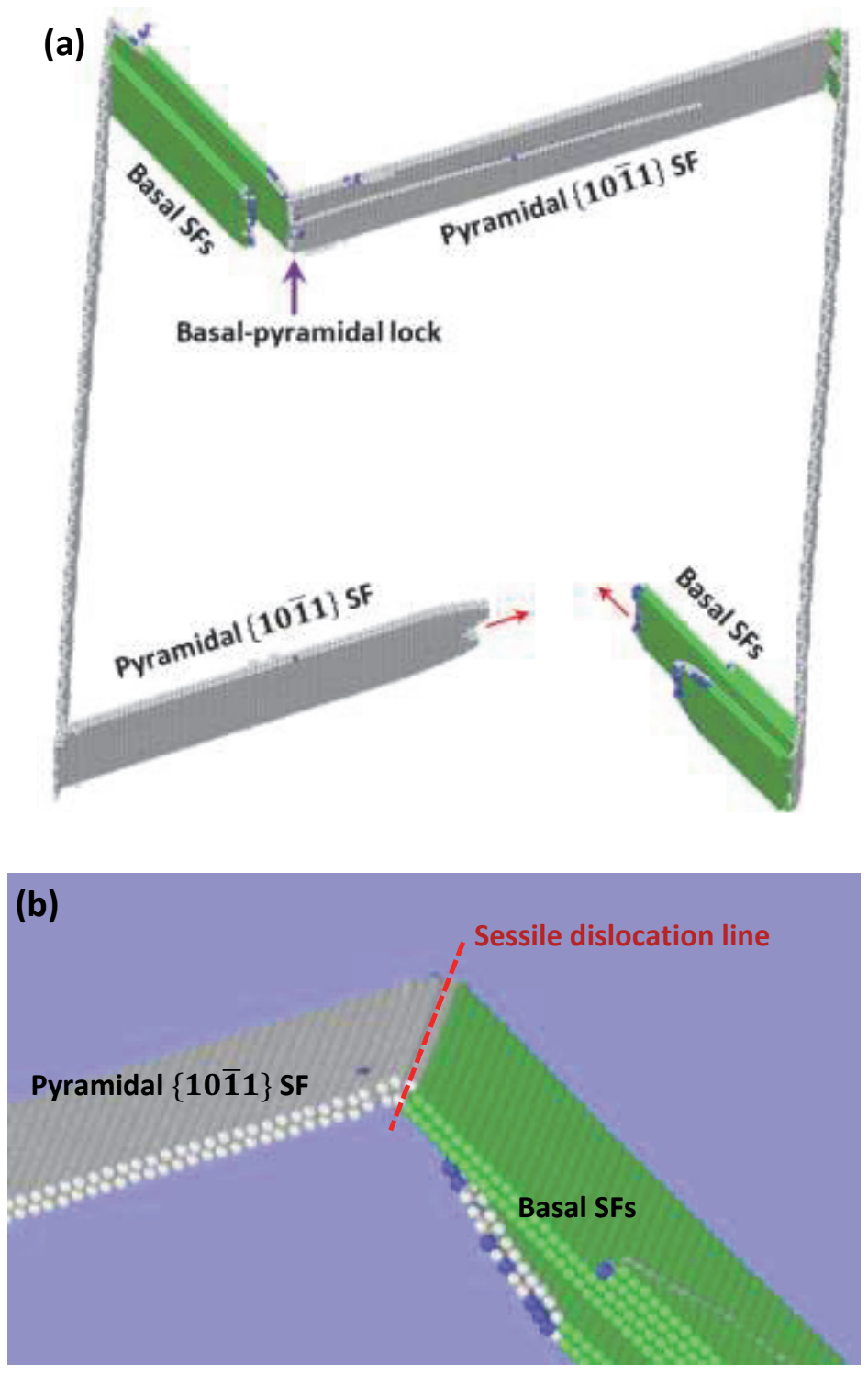

Figure 4. (a) 3-D view of the interaction between the basal and the pyramidal slip. Atoms that are located in the perfect lattice positions are removed. Atoms on the top and bottom surfaces are also removed. Leading partial dislocations bounding the SFs can be seen. After the basal SF and the pyramidal SF intersect, both slip systems are locked. (b) 3-D view of the dislocation lock which creates a sessile dislocation line along the intersection between the basal and the $\{10 \overline{1} 1\}$ plane (denoted by the dashed line). 


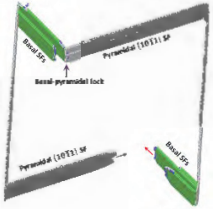

In magnesium and other hexagonal close-packed metals, the leading partial dislocations of the basal slip and the pyramidal slip meet, react and form a sessile dislocation lock, which may act as a barrier to other mobile dislocations. Such dislocation reactions create the widely observed puzzling diffraction contrast in transmission electron microscopy- 